\title{
LEP/SLC: WHAT DID WE EXPECT? WHAT DID WE ACHIEVE? A VERY QUICK HISTORICAL REVIEW
}

\author{
D.Treille \\ CERN, Genève, Suisse
}

\begin{abstract}
After a brief and personal account of LEP/SLC achievements, I recall the landmarks of their history, extending over the last 25 years. In particular I describe some attempts made at LEP1, like the use of longitudinal polarization and of a multibunch scheme. I compare the final results of LEP electroweak measurements and their accuracy to what was anticipated in various prospective studies, with the conclusion that LEP did always much better than expected. Focussing on Higgs search, I review the main steps of this adventure and confront what was achieved to what one could have done.
\end{abstract}

Invited talk at Siena 2001, Seventh Topical Seminar on The legacy of LEP and SLC. 


\section{Introduction}

Twelve years of LEP/SLC physics have brought outstanding achievements. The LEP1 era allowed to record 17 million $\mathrm{Z}^{0}$ from which most accurate tests of the Standard Model have been performed. The SLD experiment at SLC collider has measured the $\mathrm{Z}^{0}$ spin asymmetry, $\mathrm{A}_{\mathrm{LR}}$, considered as the "queen" of all electroweak observables. At LEP200 a careful exploration of all topologies and several accurate measurements have been performed up to a center-of-mass energy of $206 \mathrm{GeV}$, thanks to a "tour de force" of LEP machine physicists. These results will be reviewed in great detail during this workshop and I will only summarize them here.

However, historically, a lot of thoughts and work were devoted to some aspects of the LEP program, which, because of the priorities chosen, were not finally pursued. It is nevertheless quite instructive to review them: I will focus on the LEP1 polarization and high luminosity programs.

Furthermore, because of the pressure of the LHC project, a thorough exploitation of LEP200 potential at the highest energies could not be performed. As we will see, this would have been vital to get a pertinent answer, positive or negative, about the validity of the Minimal Supersymmetric Standard Model (MSSM), the most popular and studied prototype of model beyond the SM.

\section{A personal selection of achievements}

Avoiding to summarize the meeting in advance, I will only quote a personal choice of some of these breakthroughs.

Of the three basic entries of the SM, the $\mathrm{Z}^{0}$ mass has been measured to $23 \mathrm{ppm}$ at LEP1 (figure 1), by using a resonant depolarization method.

This was a modern epic, made possible by a close collaboration between experimen-

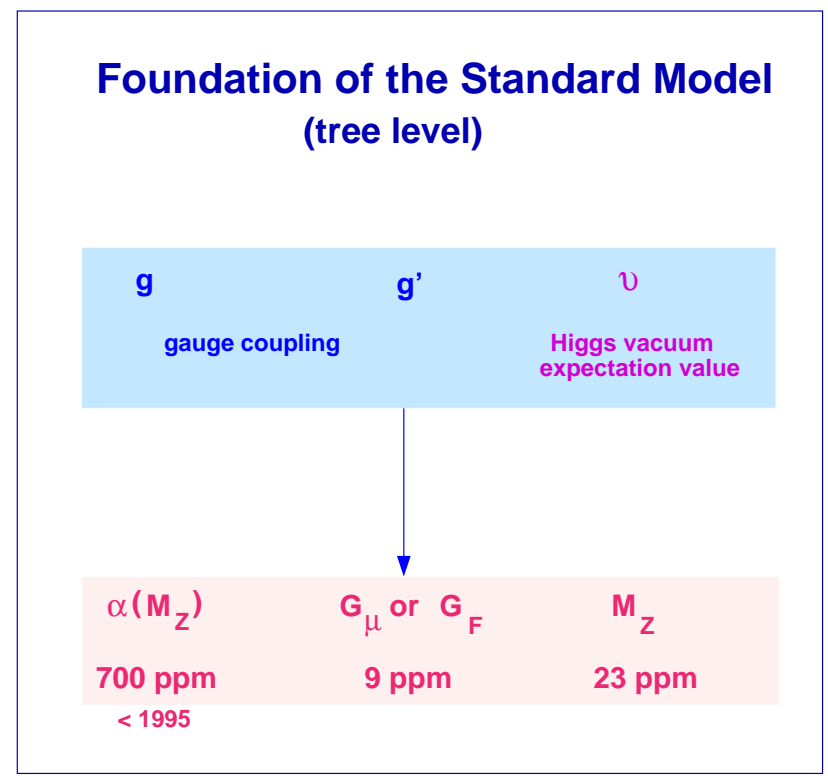

Figure 1: The three entries of the SM at tree level. 
talists and machine physicists. This accuracy compares well with our knowledge of $\mathrm{G}_{\mu}$ (9 ppm). But, unfortunately, the fine structure constant, evaluated at the relevant mass scale, is much more poorly known. In 1995 the uncertainty was $700 \mathrm{ppm}$. Thanks to various improvements it is now about $300 \mathrm{ppm}$. New theoretical inputs are welcome, but it is also mandatory to obtain a better measurement of $\mathrm{e}^{+}-\mathrm{e}^{-}$to hadrons at low and intermediate energies [1].

The $\mathrm{W}$ boson mass (figure 2) is presently known to 0.4 per mil, mostly thanks to LEP. The direct value agrees, but not too well, with the indirect one, drawn from all other measurements.

The history plots of many electroweak quantities show dramatic improvements during

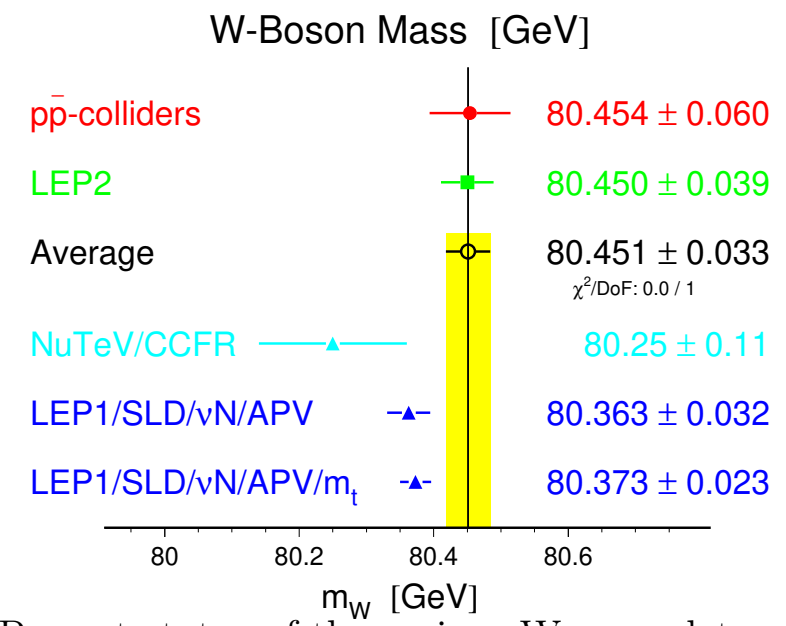

Figure 2: Present status of the various $\mathrm{W}$ mass determinations.

the LEP era. For the number of neutrinos (figure 3) and for the Weinberg angle (figure 4) [2] the accuracy was improved by two orders of magnitude.

The number of neutrinos is measured slightly below $3,2 \sigma$ away. As for $\sin ^{2} \theta_{W}$, there

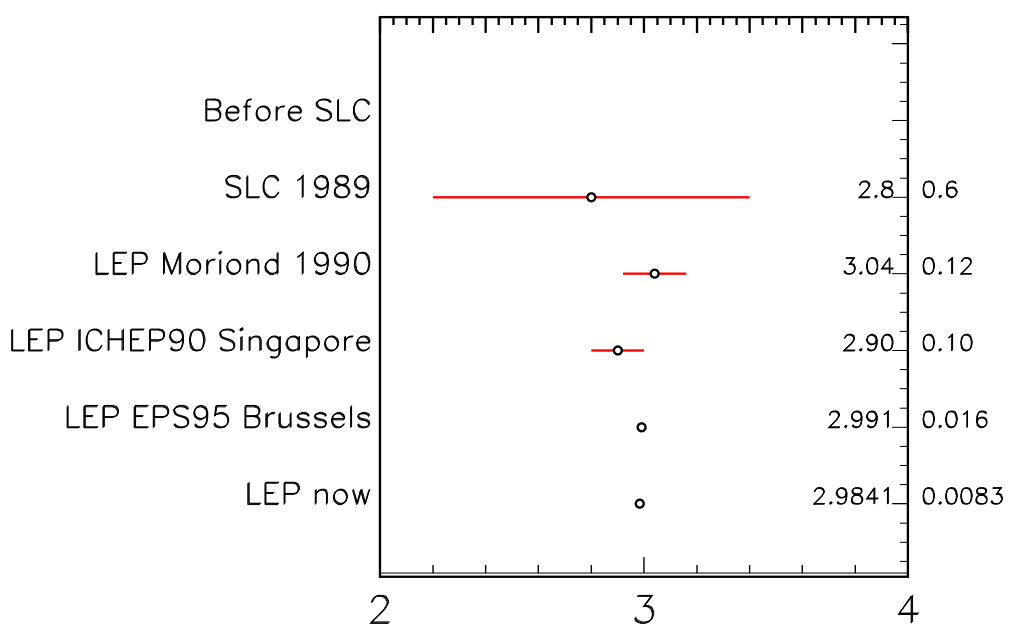

Figure 3: Evolution of our knowledge of the number of neutrinos during the LEP era.

is a persistent discrepancy between its two most accurate determinations, through $\mathrm{A}_{\mathrm{LR}}$ and $\mathrm{A}_{\mathrm{FB}}^{b}$ : it amounts to $3.2 \sigma$ by the end of LEP. 


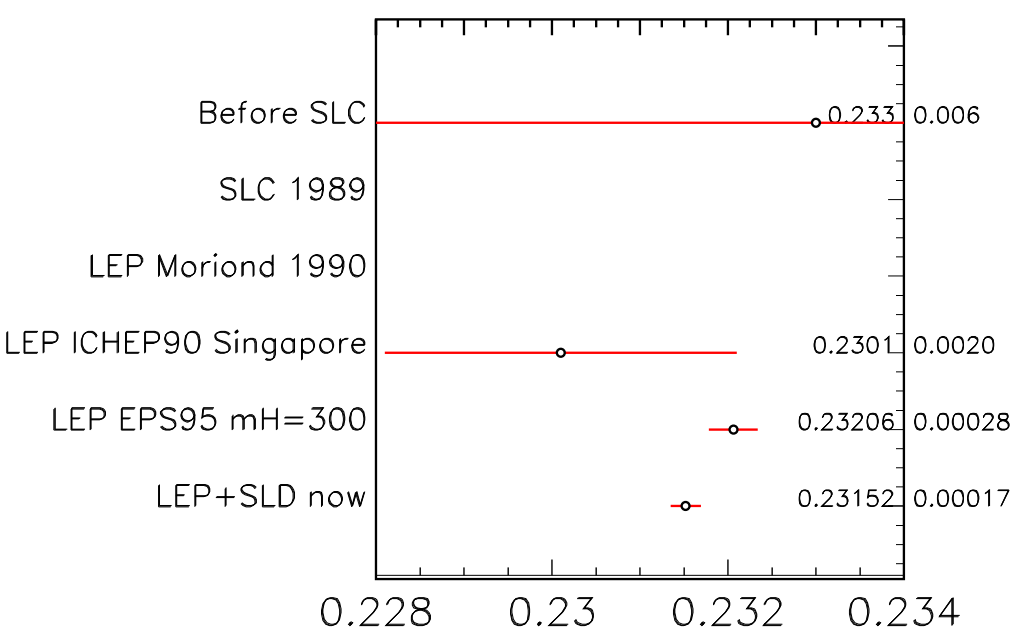

Figure 4: Evolution of our knowledge of $\sin ^{2} \theta_{W}$ during the LEP era.

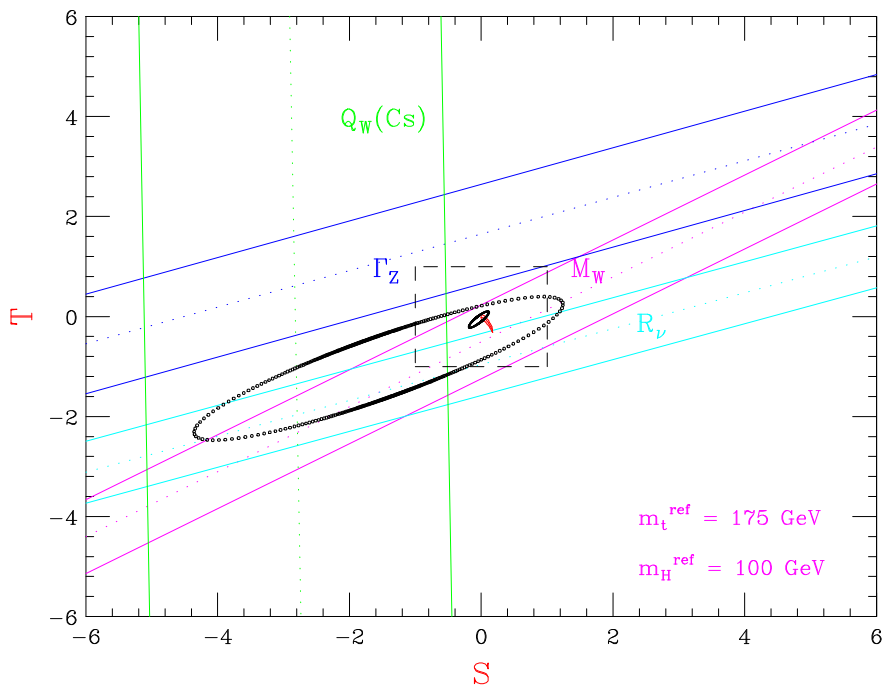

Figure 5: The information in the $\mathrm{S}-\mathrm{T}$ plane before and after LEP.

The progress brought by LEP is summarized by figure 5 [3] and figure 6 , showing the situation in the $\mathrm{S}-\mathrm{T}$ plane. Our knowledge went from the large to the tiny ellipse of figure 5. Zooming on the latter, one sees in figure 6 that the relevant information comes mostly from $\sin ^{2} \theta_{W}, \mathrm{M}_{W}$ and the $\mathrm{Z}^{0}$ lineshape.

Through the radiative corrections, LEP1 has rapidly shown that the top was heavy (figure 7) [4].

Since 94 Fermilab performed the direct measurement of the top mass. Presently the results are:

$$
\begin{aligned}
& \mathrm{M}_{\mathrm{top}}^{\mathrm{ind}}=179+13-10 \mathrm{GeV} \\
& \mathrm{M}_{\text {top }}^{\text {dir }}=174.3 \pm 5.1 \mathrm{GeV}
\end{aligned}
$$

a most remarkable agreement indeed.

The notorious figure 8 indicates that, within the SM scheme, the Higgs boson seems to be light: $\leq 196 \mathrm{GeV}$ at 95 percent confidence level.

Two obvious questions are then the following ones:

- does this result represent the truth, or is it the effect of a conspiracy, an eventuality which is not very natural but nevertheless possible ${ }^{[5]}$ ? 


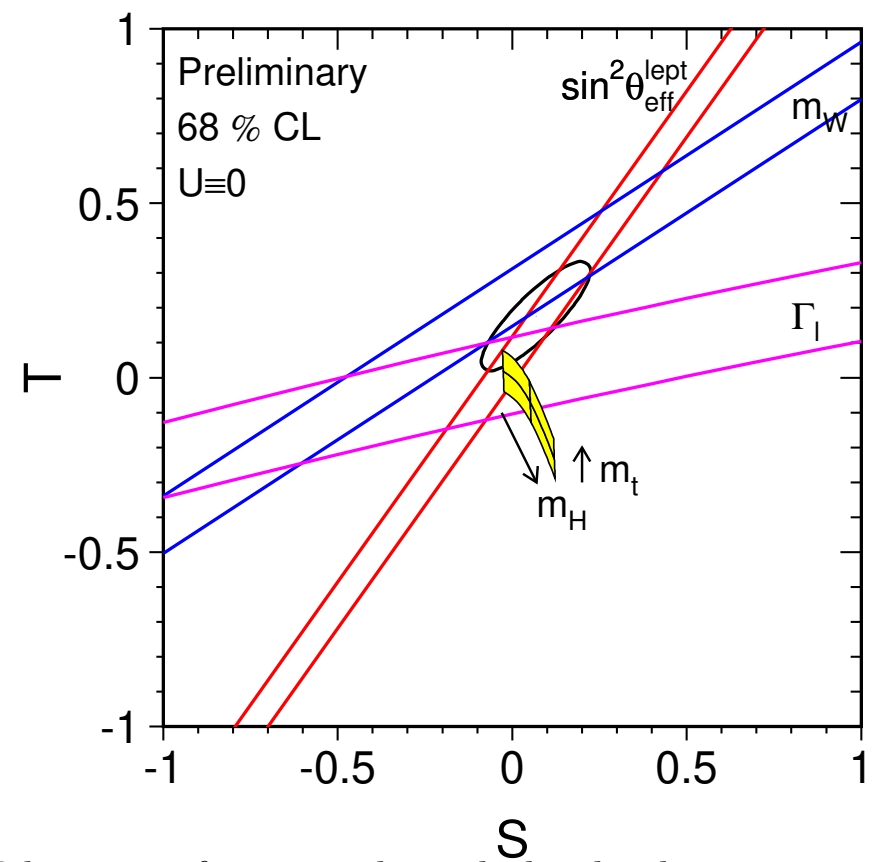

Figure 6: The LEP legacy: information brought by the three most performant observables.

- among the somewhat discrepant values of $\mathrm{M}_{H}$ provided by the different observables (figure 9) [6], which is the correct one?

A very striking result from LEP1 is certainly the demonstration of the excellent convergence of the couplings in SUSY, as shown by figure 10 [7]. On the contrary, in the $\mathrm{SM}$, such a convergence does not occur, and concerning this discrepancy LEP accuracy has transformed a $2 \sigma$ problem into a $8 \sigma$ one.

LEP1 has shown that the $\tau$ is a well-behaved lepton, mere recurrence of the first two, except for its mass. Figure 11 [8] shows how LEP (tau lifetime and leptonic branching ratio), BESS (a beautifully accurate mass measurement) and CESR (leptonic branching ratio) have clarified the situation in a truly international venture. The tau being "normal", one can then use it as a most interesting laboratory for hadronic physics and QCD.

B physics at LEP1 has been a very active area. I will simply summarize its achievements by figure 12 [9], which shows the progress in our knowledge of the tip of the Unitary Triangle, mostly but not entirely due to LEP.

Finally the outstanding contributions of LEP to QCD, in particular to a variety of $\alpha_{S}$ measurements, are well known and will be abundantly described.

As for LEP200, in the field of SUSY, it set a lower limit on the masses of many predicted species up to $\sim \sqrt{s} / 2$ and excluded the existence of an LSP (lighest supersymmetric particle) up to $\sim 45 \mathrm{GeV}$.

\section{LEP history in a nutshell}

The idea of building a high-energy electron-positron colliding beam storage ring appeared in a note by B.Richter [10], being then a visitor at CERN, in march 1976.

Experimental possibilities were described, a cost minimization technique was developed 


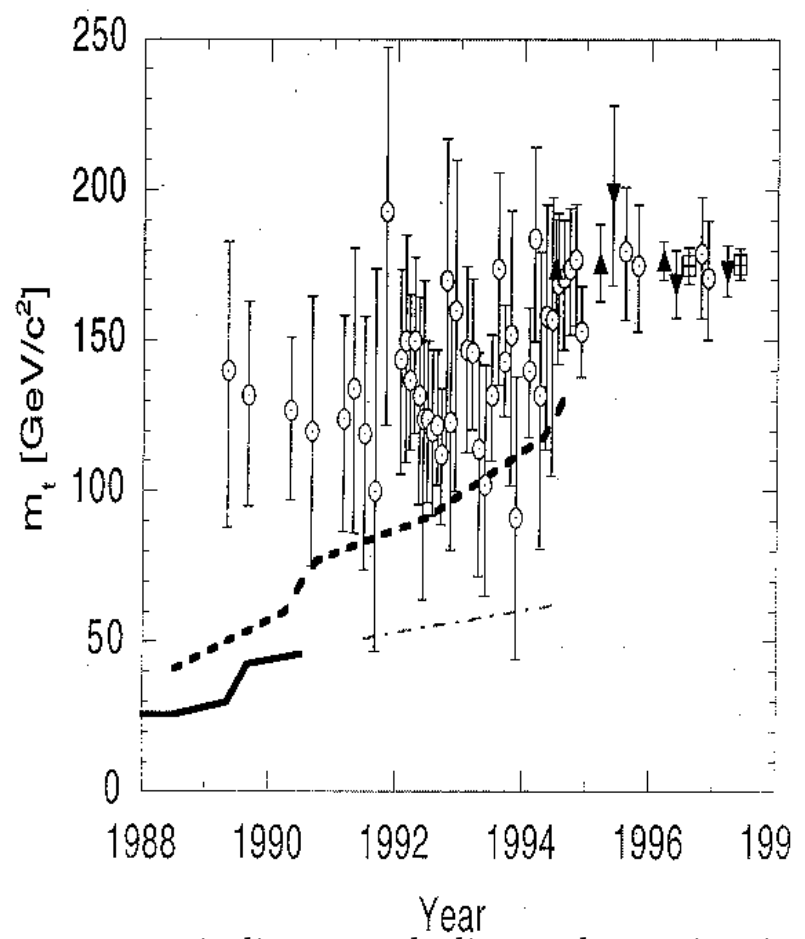

Figure 7: The top mass: indirect and direct determinations along the years (from C.Quigg).

and a model machine designed to operate at center-of-mass energies of up to $200 \mathrm{GeV}$. The rationale for this last figure is summarized in figure 13, which tells that basically the LEP scenery was correctly anticipated.

Figure 14 [11] gives a guess of the respective costs of a circular machine and a linear collider. It shows that the cross-over occurs roughly at the top of LEP range: it is clear however that once LEP was built there was a "window of opportunity" for this machine in this energy region, which was calling for a more accurate definition of its maximum energy depending on physical arguments.

Due to financial, geological and political arguments, the LEP circumference and its maximal beam energy kept oscillating for some time [12]. In the Blue Book of 1978 they were $22.2 \mathrm{~km}$ and $100 \mathrm{GeV}$, in the Pink Book of $197930.6 \mathrm{~km}$ and $130 \mathrm{GeV}$, and finally in the LEP Design Report of $198426.6 \mathrm{~km}$ and $125 \mathrm{GeV}$.

In 1978 an important prospective meeting was held in les Houches [13]. In his most interesting summary talk, S.Glashow expressed his views on the situation:

"Since the low energy limit is so well confirmed, few can doubt the truth (of the by-now "Standard" model)."

But he considered then four possible scenarios. For him "the least probable" was the 17 parameters scenario, i.e the SM:

"It would be both arrogant and unhistorical to believe that our naive extrapolation from physics at $2 \mathrm{GeV}$ to physics at $200 \mathrm{GeV}$ is likely to be correct in detail".

On the contrary he thought the "most likely" would be a scenario leading to a complete surprise.

Figure 15 gives the planning of the LEP project as it was presented in les Houches by J.Adams. 


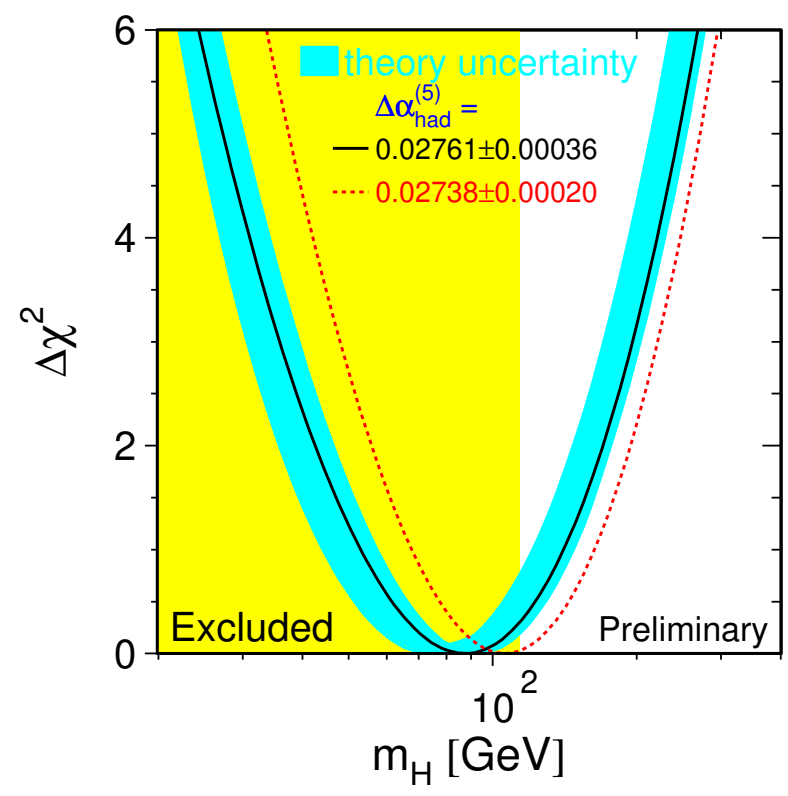

Figure 8: The indirect information on the Higgs mass from accurate electroweak measurements.

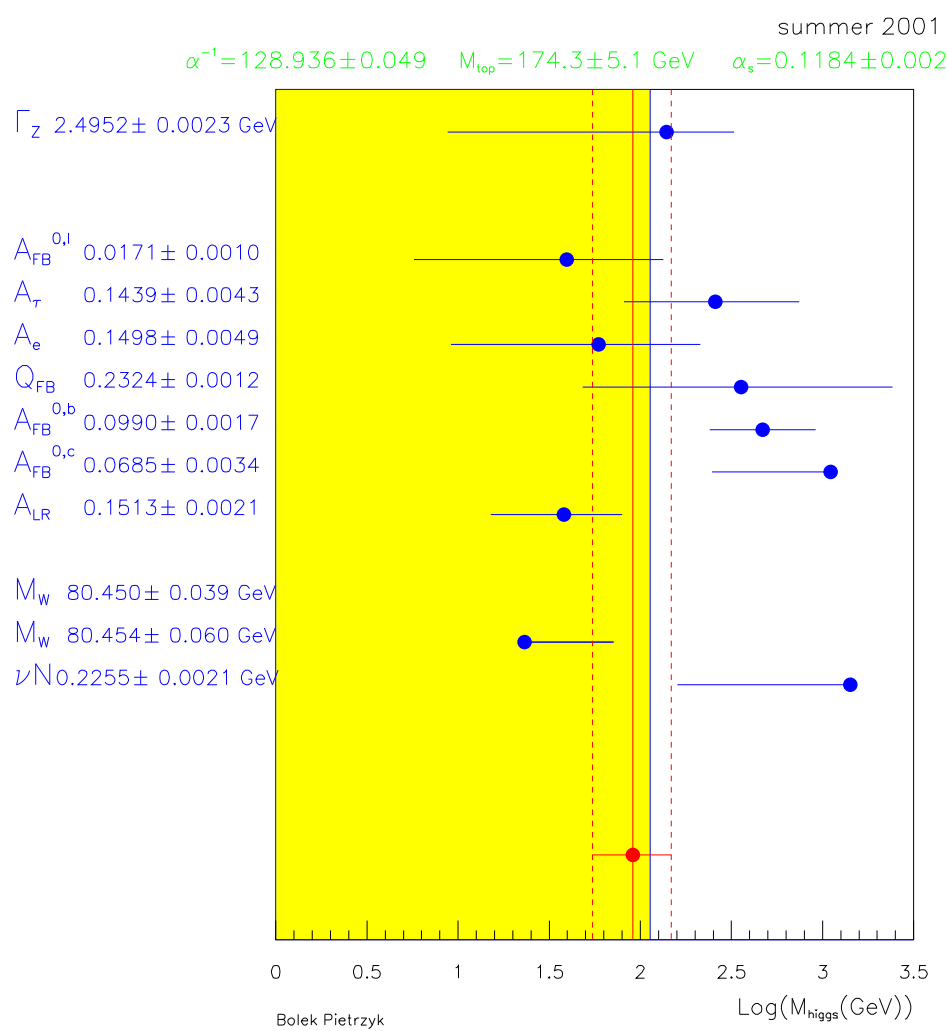

Figure 9: The indirect information on the Higgs mass from each observable (from B.Pietrzyk). 


\section{Unification of the Coupling Constants in the SM and the minimal MSSM}
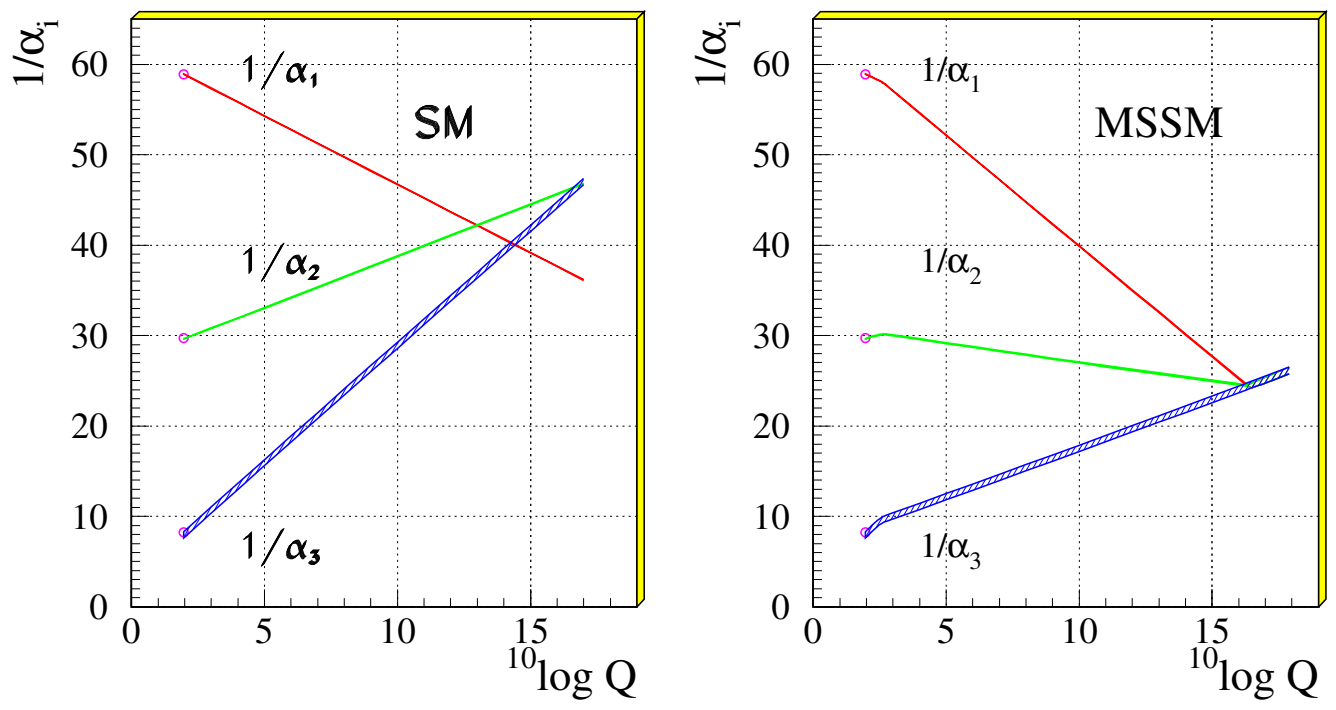

Figure 10: The unification of couplings in the SM and in SUSY.

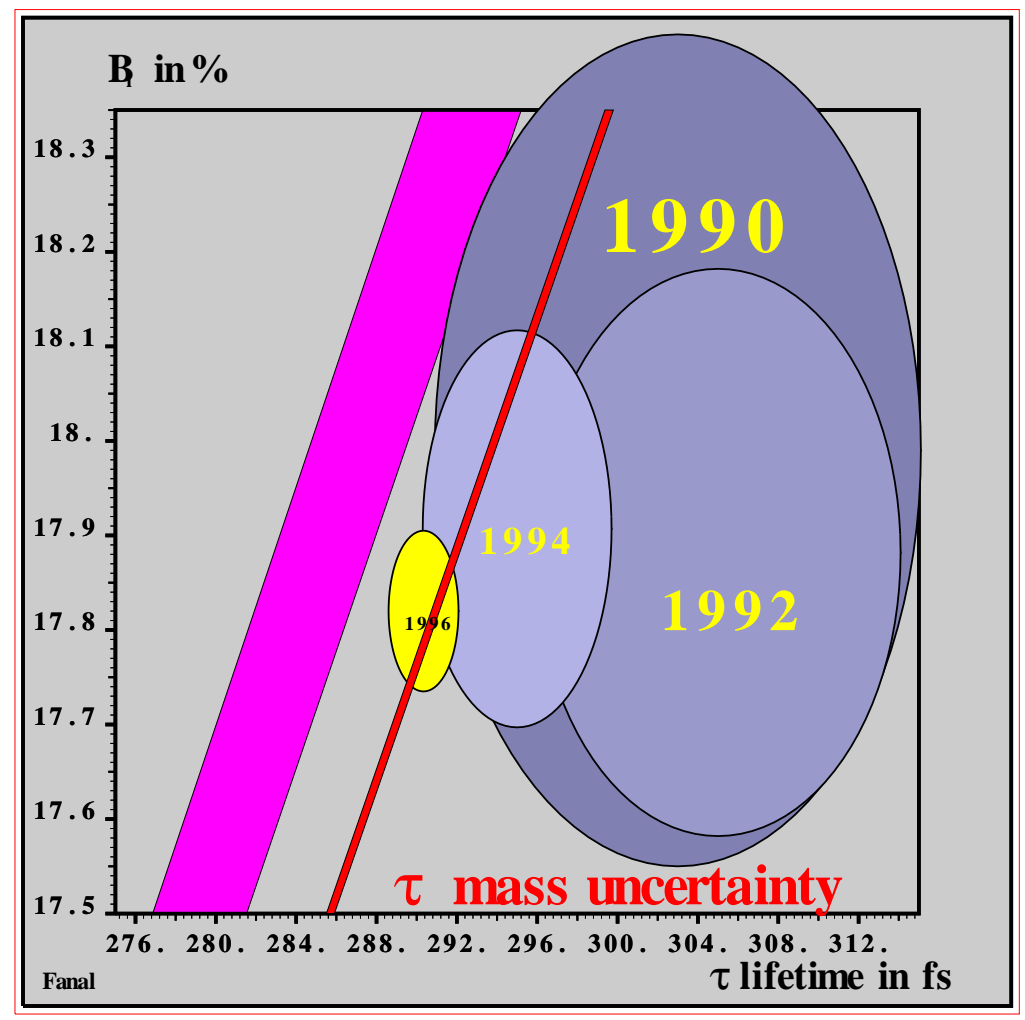

Figure 11: The tau is normal. 

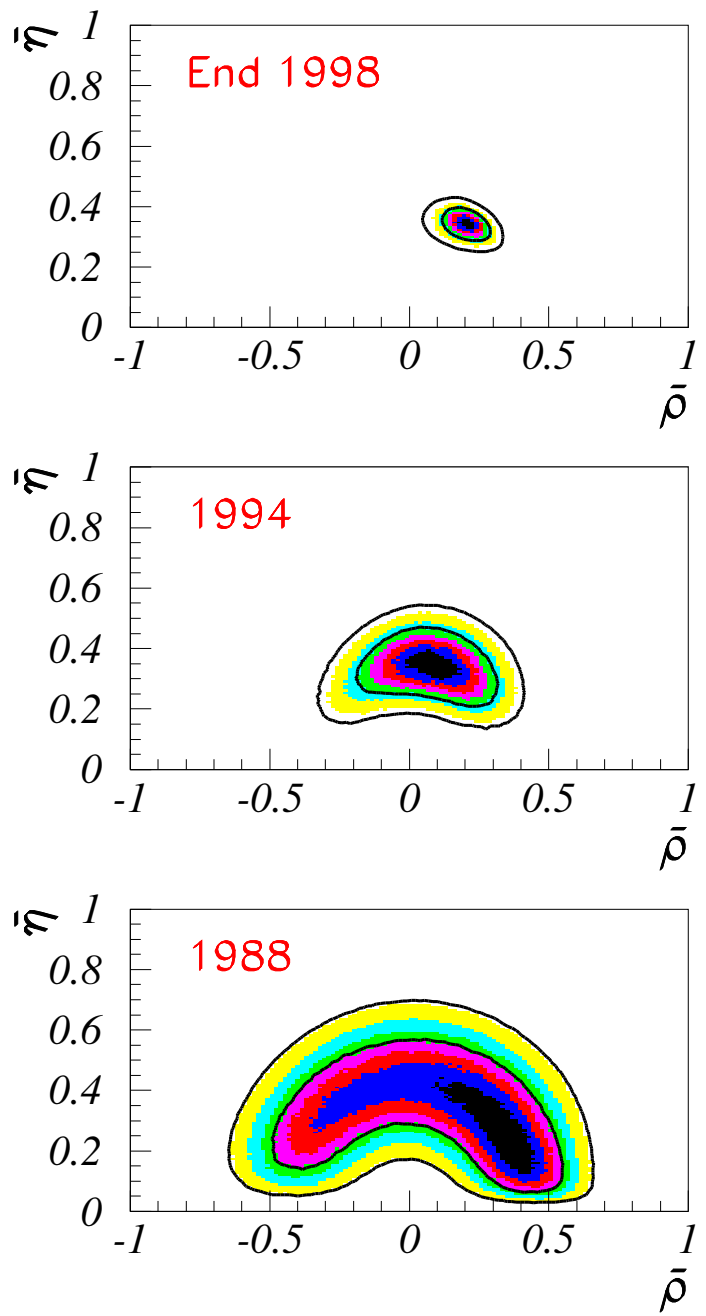

Figure 12: The progress in B physics during LEP era.

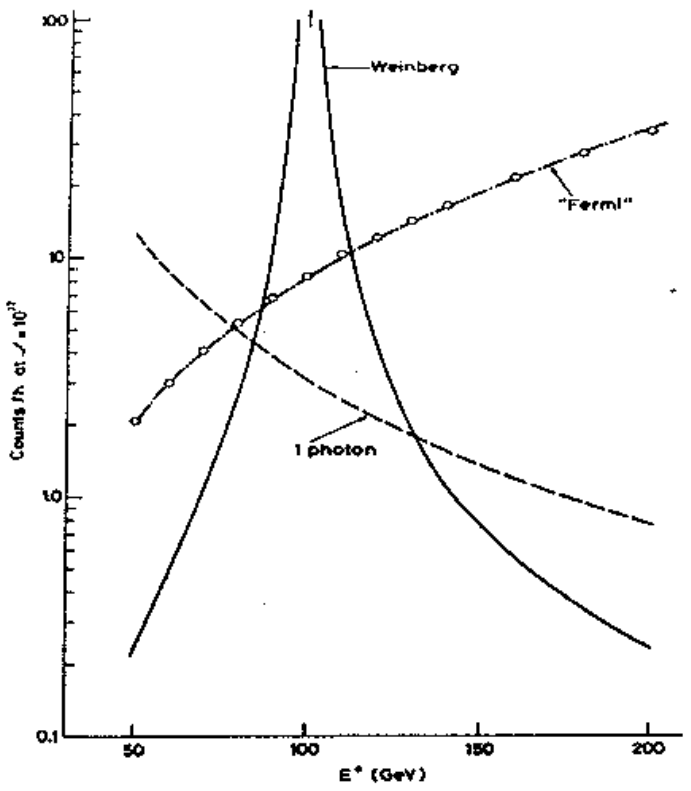

Figure 13: The possible LEP scenarii as presented by B.Richter in 1976. 


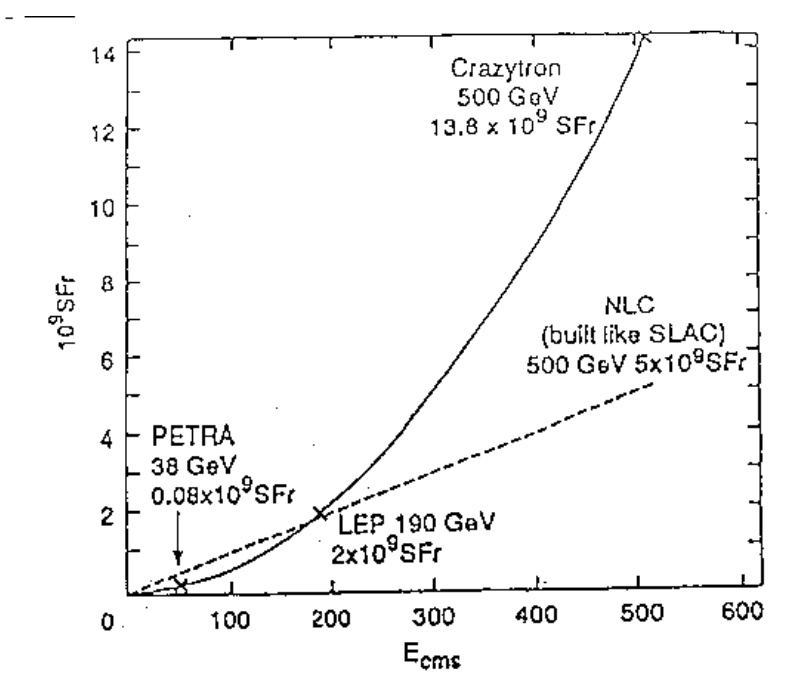

Figure 14: Guess of the costs of a circular and of a linear $\mathrm{e}^{+}-\mathrm{e}^{-}$collider versus energy.

The 1979 Nobel Prize was attributed to S.Glashow, A.Salam and S.Weinberg for their contributions to the Standard Model.

In 1982 the LEP project got its final authorization, with the unconditional support of all Member States. Meanwhile the LEP team had continued to work on the optimization of the designs and on the exact location of the ring. It had decided to tilt the plane of the ring. Active $\mathrm{R} / \mathrm{D}$ work on the RF cavities, warm and supraconducting, was already under way. The LEP management Board, with E.Picasso as Chairman, had been set up, as well as the LEP Experiments Committee, under G.Wolf of DESY, and a LEP Machine Advisory Committee, under G.A.Voss of DESY.

In 1983 the Déclaration d'Utilite Publique for the LEP machine was signed, and the civil engineering could start. Collisions were then foreseen for the second half of 88 . The same year the $\mathrm{Z}^{0}$ was discovered at some $92 \mathrm{GeV}$, the mass predicted by theory, well within LEP's grasp.

1986 was the year of an important Workshop at Cern, on Physics at LEP [14]. The foundations of the program of accurate tests of the electroweak theory were laid [15] and the experimental methods defined in already great detail. However we were still in the dark concerning the top mass, and a lot of work was devoted to scenarios where the $t-\bar{t}$ threshold bound states was close to the $\mathrm{Z}^{0}$, and even degenerate with it.

By the mid-eighties ideas about the "after-LEP', namely the LHC (17 TeV, $10^{33}$ $\mathrm{cm}^{-2} \mathrm{~s}^{-1}$ ), were quite clearly defined [16]. The $10^{34}$ high luminosity option appeared in 1987 after the la Thuile ECFA meeting [17].

In february 1988 the LEP tunnel excavation was completed. In july 1989 the first $Z^{0}$ were registered in the four experiments. You all know the remaining of that exciting story. 


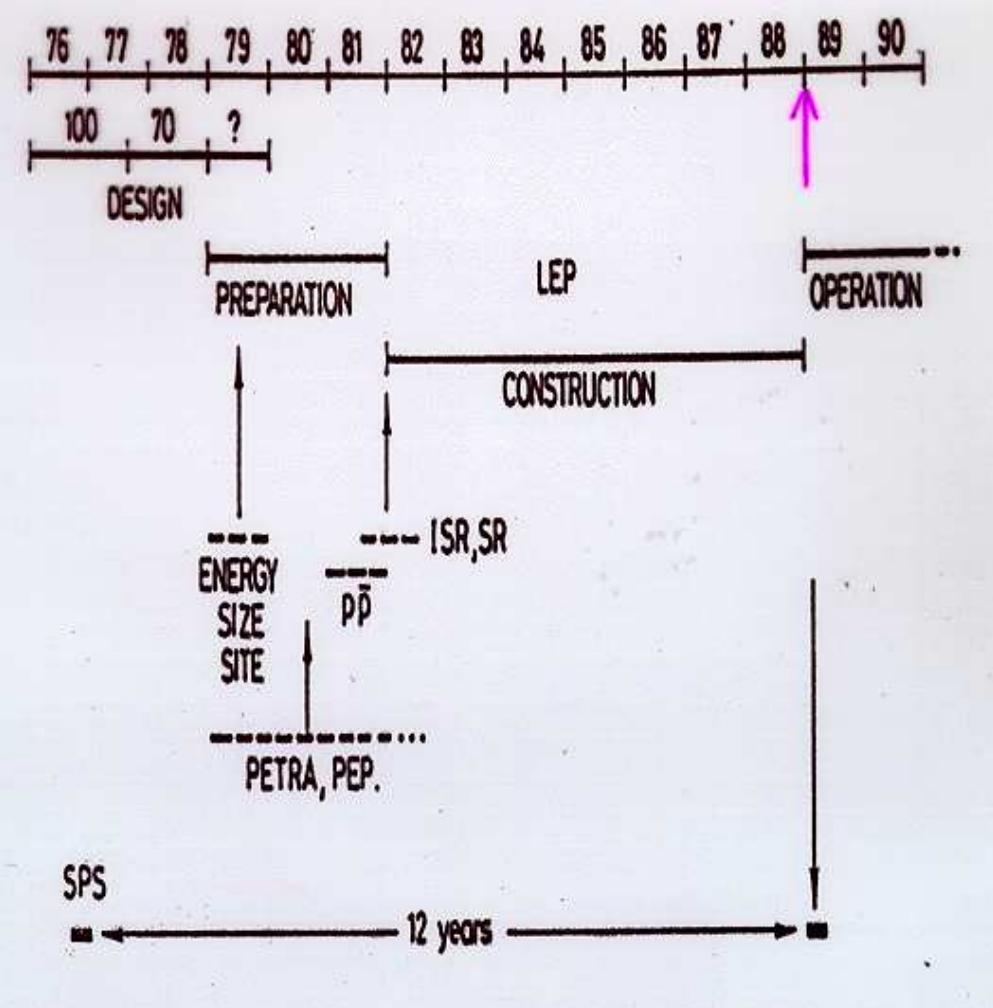

Figure 15: J.Adams's LEP planning in les Houches 1979.

\section{A brave attempt: Polarization at LEP}

It is well known that spin is at the heart of the Standard Model. The potential impact of longitudinal polarization on electroweak tests was fully recognized [18] and a Workshop held in 1988 [19] studied the feasibility of such a program for LEP.

In a circular machine there is a natural build-up of transverse polarization, due to the Sokolov-Ternov effect [20]. However this is a slow process and various depolarization mechanisms counteract it. One must therefore speed up the rate of its growth and maintain the degree of polarization achieved: the latter implies a careful "spin matching" of all components of the machine. Finally one has to rotate the polarization from transverse to longitudinal. Without any further action the result would be disastrous since one would obtain the same helicity in both beams and zero cross-section for full polarization: one must therefore use the selective depolarization of one beam.

Actually a very clever scheme was then invented by A.Blondel [21] (see part d of figure 16), in which, through selective depolarization of some bunches, one achieves four different types of crossings. The first two give the left-right asymmetry one is looking for, while the last two give the amount of polarization of the beams by a simple $\mathrm{Z}^{0}$ counting method. In first approximation, one does not need to measure "externally" the polarization with a polarimeter, while this is a necessity in the SLC scheme where only electrons are polarized.

I cannot do justice here to the large amount of clever work put in that study, which in particular led LEP to reconsider the normalization schemes, and especially the very 


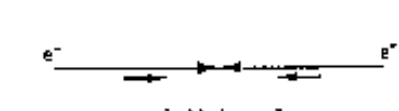

a) Natural

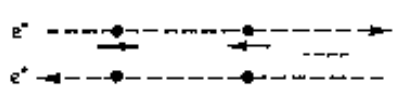

c) SLC schome

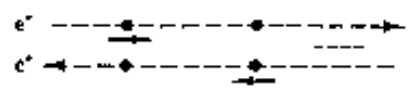

b) 2ho-bunch scheme at Lete

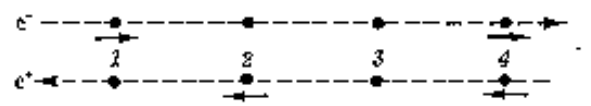

d) Four-bunch sohenc at LEP

Figure 16: The idea of the Blondel scheme.

small angle luminometers, for relative luminosity measurements. Spin matched rotators of the Richter-Schwitters type were designed in great detail [22]: their use would have forced to tilt longitudinally the experiments by $\sim 1$ degree! The corresponding increase of the synchrotron background foreseen led us to improved studies of the masking schemes at LEP which turned out to be very useful at LEP200.

The goal of a polarization measurement at LEP with the Blondel scheme was to reach an experimental error on $\sin ^{2} \theta_{W}$ of \pm 0.0003 . This would have required a million $\mathrm{Z}^{0}$, taken with a polarization of 0.5 and a luminosity relative accuracy of 1.5 per mil.

The full priority given to the energy rise at LEP finally led to discard the longitudinal polarization program, leaving thus SLC by itself to measure the $A_{\mathrm{LR}}$ asymmetry. The SLAC program was a great success. A polarization of 75 percent was achieved with a strained lattice cathode. With only $500 \mathrm{~K} \mathrm{Z}^{0}$ on tape they achieved an accuracy on $\sin ^{2} \theta_{W}$ of 0.00026 [23], better than the goal of LEP polarization program. It is however regrettable that no second measurement was available for a cross-check.

At LEP transverse polarization was abundantly exploited later to get a most precise measurement of the beam energy.

The Blondel scheme is certainly a must for any future $\mathrm{Z}^{0}$ factory. A linear collider will then have the problem to provide polarized positrons, as well as electrons. May be an ideal $\mathrm{Z}^{0}$ factory would have been a circular machine like LEP, benefiting of the Sokolov-Ternov effect, and with two rings instead of one, to be able to cope with a large number of bunches in order to reach high luminosities, without worrying about their parasitic crossings.

\section{A higher luminosity at LEP}

Another attempt was to turn LEP into a multibunch machine. The idea was to exploit a "Pretzel scheme" in which the orbits have a oscillatory shape, in such a way that the counter rotating bunches do not see each other, except at or near the collision points. A full workshop was devoted to that study [24]. Schemes involving as many as 36 bunches were considered, with the conclusion that peak luminosities beyond $10^{32}$ at the $\mathrm{Z}^{0}$ could be obtained, without any really drastic change to the machine, but with the addition of electrostatic or RF-magnetic separators, with various upgrades and a more complicated operating mode. The modifications required by the experiments were studied as well: they were minor for 8 bunches (the final outcome of the exercise) and would have been more substantial for the 36-bunch solution. 
An important result of the workshop was the evaluation of the expected evolution of the accuracy of the electroweak measurements when the $\mathrm{Z}^{0}$ exposure is increased, and to which extent systematic errors could actually be measured from the data, becoming thus statistical errors, rather than determined by Monte Carlo. This will be discussed in the next section.

During this Workshop the potential of LEP1 for tau and B physics was analysed in great detail and compared to other programs, like flavour factories. The $\mathrm{Z}^{0}$ is actually the most abundant source of beauty at $\mathrm{e}^{+}-\mathrm{e}^{-}$machines (6.3 nb compared to $1.2 \mathrm{nb}$ at an asymmetric beauty factory). As we said, the clean topology, the strong boost, as well as the fact that in a single exposure all B species are produced, are important assets. Table 6.7 of [24] describes the various possibilities and shows that a polarized $\mathrm{Z}^{0}$ factory compares favorably to the other ones at an equivalent luminosity.

Among the topics most debated let me quote the foreseeable performance of b-tagging, purity versus efficiency. Quite impressive figures were already quoted in the simulations [25]: they were still received with some scepticism, although they were much below what was finally achieved at LEP once modern microvertices were installed and well understood. Furthermore, with the promise of a high number of $Z^{0}$, double tag techniques with quite low systematic errors were introduced.

Concerning the B lifetimes, one question was whether one would be able to measure the individual values for the different species with enough accuracy. Quoting [26]:

"Can we go below the interesting level of 10 percent on individual lifetimes?"

And the guess was that ".. with $10^{7} \mathrm{Z}^{0}$, one would reach accuracies of 3 to 4 percent...".

Figure 17 giving the final LEP results shows that the prognostic was quite good.

Similarly, concerning the $\mathrm{B}_{S}^{0}$ oscillations, it was said that [27]:

"With $10^{7} \mathrm{Z}^{0}$ one can measure oscillations up to $\mathrm{x}_{S} \sim 10$. With $\sim 510^{7} \mathrm{Z}^{0}$ one can reach $\mathrm{x}_{S} \sim 15 . "$

Actually, as shown by figure 18 , the latter number was overtaken with $\sim 1710^{6} \mathrm{Z}^{0}$. On this figure one can notice a tantalizing feature of the amplitude curve, insufficient however to draw a firm conclusion on a possible oscillation.

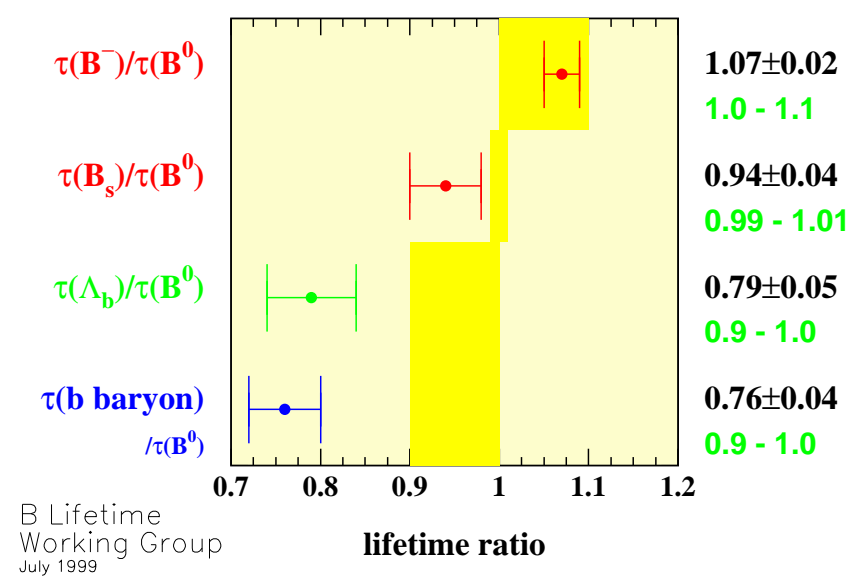

Figure 17: Our present knowledge of the individual B lifetimes. 


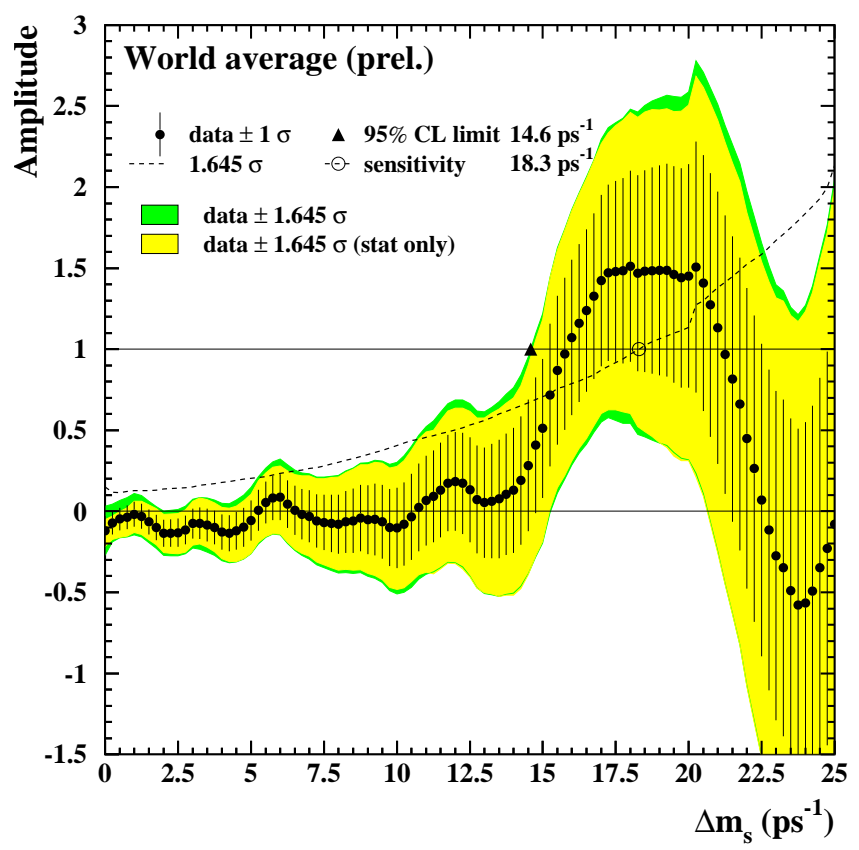

Figure 18: Present limits on Bos oscillations.

\section{Electroweak measurements: expectations and achievements}

It can be amusing and instructive to focus retrospectively on what was expected from LEP before the program was started. Such prospective exercises were done in particular in 1988, at the occasion of the Polarization Workshop [28], and later, in 1990, in the LEPC Cogne meeting during which the priorities of the LEP program were defined.

Starting with the $\mathrm{Z}^{0}$ mass measurement, in 1988 the ideas of an optimized scan and of using spin-resonance energy calibration were present. The foreseen statistical error was $\pm(10-15) \mathrm{MeV}$, while the systematic one was $\pm 17 \mathrm{MeV}$, with equal contributions from the stability of the luminosity monitor, the systematics from QED corrections and the energy uncertainty. As you know, LEP finally achieved an overall uncertainty of 2.1 $\mathrm{MeV}$, one order of magnitude better.

For the $\mathrm{Z}^{0}$ width a systematic error of $\pm 50 \mathrm{MeV}$ dominated by the normalization error was expected: the final result was $2.3 \mathrm{MeV}$ overall.

When Feldman [29] proposed his method for measuring $\Gamma_{i n v}$, the foreseen relative uncertainty on the luminosity was still 3 percent (and this was considered as optimistic...) and the relative uncertainties of the acceptance of various channels ( $\mu \mu$, hadronic) were at the percent level.

Finally LEP achieved 1 per mil for the former (0.6 per mil for theory) and 1 per mil for the latter. The spectacular result concerning the absolute normalization is also clearly part of the explanation of the performance for $\mathrm{M}_{Z}$ and $\Gamma_{Z}$.

As for the measurement of the $\mathrm{W}$ mass, the methods which were finally used had been accurately studied already in the Aachen 1986 Workshop, that we will review later: the emphasis was correctly put on the $\mathrm{W}$ reconstruction method and the sources of uncertainty rightly identified, although there too the anticipated errors were on the pessimistic side. "Each of the four LEP experiments can measure in at least three ways 
the mass of the $\mathrm{W}$ boson at LEP200 with an accuracy of the order of $100 \mathrm{MeV}$ or better. The integrated luminosity of $500 \mathrm{pb}^{-1}$ used in this study provides a better statistical accuracy $(50-60 \mathrm{MeV})$ but it appears difficult to control the systematical uncertainties at such a level."

Concerning asymmetries, as we said, the SLC, through $A_{L R}$ and with only half a million $\mathrm{Z}^{0}$, measured the Weinberg angle better than what we anticipated from the polarization program at LEP.

For $\mathrm{A}_{c h}^{\mu \mu}$ the expected systematic error was 0.18 (about equally due to the uncertainty on the $\mathrm{Z}^{0}$ mass, on the detection efficiency and on the QED radiative correction). LEP did twice better.

Similarly for the $\tau$ polarization: the utilisation of the $\rho \nu$ channel, not foreseen initially, and an excellent control of the tau final states, led to an accuracy on $\sin ^{2} \theta_{W} 2.5$ times better than anticipated.

Concerning heavy quarks measurements, once introduced the idea of double tag, the relative importance of systematic and statistical errors was correctly anticipated. For $\mathrm{R}_{b}$, compared to the estimate of ${ }^{[30]}$, the accuracy achieved is a factor 3 to 6 times better. From $\mathrm{A}_{\mathrm{FB}}^{\mathrm{b} \overline{\mathrm{b}}}$, the accuracy on $\sin ^{2} \theta_{W}$ is three times better (0.0009 anticipated, 0.00031 achieved, the statistics dominating as expected). However the absolute value of $\sin ^{2} \theta_{W}$ from $A_{\mathrm{FB}}^{\mathrm{bb}}$, as you remember, is at variance with the one from $A_{\mathrm{LR}}$, and we are left with this question mark.

At the 1990 Cogne meeting one exercise was to compare the accuracy on $\sin ^{2} \theta_{W}$ as expected from the unpolarized asymmetry measurements and their combination to what the LEP polarization program could give. This is summarized by figure 19 [31]: it shows that 25 million $\mathrm{Z}^{0}$ would be equivalent in this respect to the polarization program. The choice was then to discard the latter and to require 4.5 million $\mathrm{Z}^{0}$ per experiment before leaving the peak to raise the energy towards LEP200, considered as the main priority after LEP1. Soon after Cogne, the practice to combine results from all four experiments was started, first in the field of electroweak measurements.

In summary, all achievements were better, often much better, than foreseen. This is due to a number of facts:

$1 /$ the outstanding performances of the machine.

2/ the relative easiness of LEP physics: with such a low level of background the interesting events were easy to select and very clean. Clever masking schemes [32] allowed even to decrease the radius of the vacuum chamber, a most beneficial step for b-tagging performances.

$3 /$ the redundancy of all experimental procedures. This, for instance for the trigger, had been correctly anticipated and planned: as a result any efficiency could always be measured instead of being evaluated from MC, so that important systematic errors were actually turned into statistical ones.

4/ the development of new techniques, some appearing actually during LEP lifetime. The most important were the conception of extremely accurate small angle luminometers [33] and of highly performant, multilayer, double-sided microvertex detectors [34].

$5 /$ the high quality of theoretical estimates.

Furthermore full sectors of physics, like tau physics and beauty physics, could be thoroughly covered, much better than foreseen: LEP1 was thus a good first approximation of a $\mathrm{Z}^{0}$ factory, hence of a tau and $\mathrm{B}$ factory. Here again the most favourable topology of the events, with two back-to-back well separated jets, the strong boost of heavy objects and 


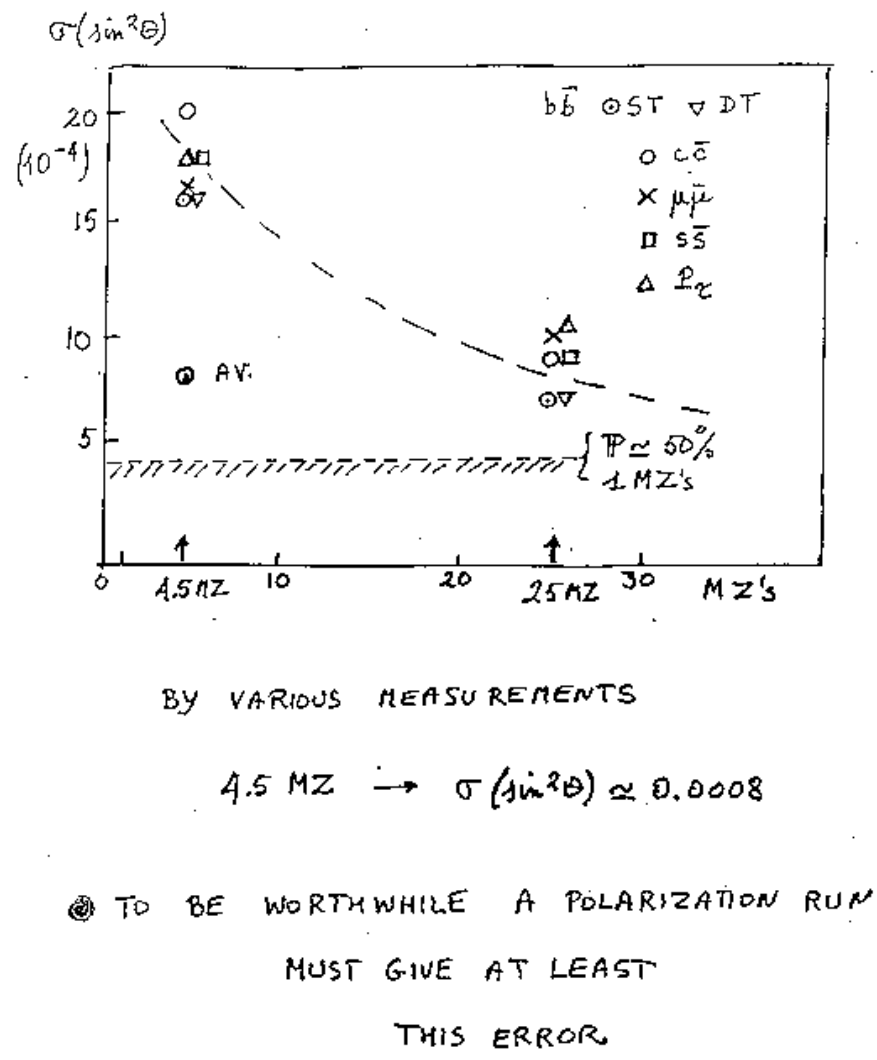

Figure 19: Foreseen accuracies of electroweak measurements versus the number of $\mathrm{Z}^{0}$. From Ugo Amaldi.

the high quality of the detectors for microvertexing and identification were the main assets.

\section{The Higgs boson search: a quick survey}

I will start with the Aachen 1986 LEP200 Workshop. The prospects concerning supraconducting cavities were very clearly presented [35]. Among other physics topics, the Higgs search was studied in some detail [36]. However, at that time, it was still considered as impossible, for experimental reasons, to explore the domain of Higgs mass in the vicinity of the $\mathrm{W}$ mass, and a fortiori of the $\mathrm{Z}$ mass.

From the Higgs study group presentation one reads:

"..the conclusion is that, at center-of-mass energy of $200 \mathrm{GeV}$ and with an integrated luminosity of $500 \mathrm{pb}^{-1}$, significant signals are certainly observable up to $\mathrm{M}_{H}=80 \mathrm{GeV}$ from the missing energy channel and up to $\mathrm{M}_{H}=70 \mathrm{GeV}$ from the 4 jet channel."

And from the Workshop Summary:

"Clearly the 4 jet events are not useful if $\mathrm{M}_{H}=80-90 \mathrm{GeV}$ since there could be complete confusion with WW and ZZ production,..."

Similarly later:

"LEP200 should detect the Higgs if $40 \leq \mathrm{M}_{H} \leq 80 \mathrm{GeV}$, but it will be difficult. It is not at all clear that hadron colliders (of any energy) would be able to detect the Higgs at all." 


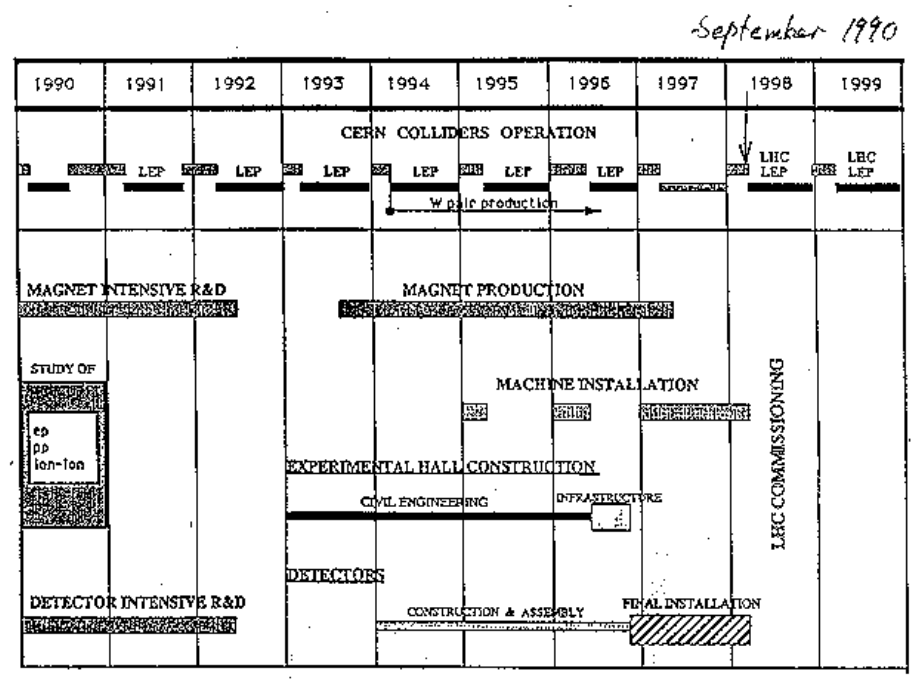

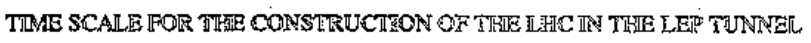

Figure 20: The LHC planning foreseen at the time of Cogne meeting.

In 1989 however it started to be realized that with a good b-tag one would probably be able to "break the $\mathrm{Z}^{0}$ wall". Quoting from [37]:

"..If the mass was higher, i.e. in the vicinity of $\mathrm{M}_{Z}$, then it was felt that the ee $\rightarrow$ ZZ background would preclude observation of the Higgs. However, if an efficient tagging of the b $\bar{b}$ final state is performed, this may not be the case... More study is needed - and is worthwhile- since the presence of a scalar in the vicinity of $\mathrm{M}_{Z}$ has often been advocated. This would again be an argument in favour of effectively reaching $\sqrt{s}=200$ GeV at LEP."

On the basis of simple estimates, the rule of thumb for a reach of: $\mathrm{M}_{H} \sim \sqrt{s}-100$ $\mathrm{GeV}$ was proposed, with the meaning of a discovery potential per experiment.

Many subsequent Monte Carlo studies were performed along the years to validate that rule, and to improve it progressively by combining the four experiments and introducing more sophistication in the analysis and statistical methods.

In 1991 appeared the preliminary computations of the radiative corrections to the mass of the lightest MSSM Higgs boson $\mathrm{h}^{0}$ [38], showing that they could be large and that the exact value of the top mass was a critical ingredient since it enters there to the 4 th power.

In 1992 a first LEP2 LEPC Workshop was held at Cern. In its report, the Higgs and SUSY Higgs Working Group wrote in particular:

"It was also shown that, if the top mass is not too high $(\sim 140 \mathrm{GeV})$, it is worthwhile studying the possibility of going to a higher $\mathrm{cm}$ energy, since an increase to $210 \mathrm{GeV}$ could allow the exploration of the quasi-totality of the parameter space of Minimal SUSY."

From that Workshop stemmed the now familiar $\mathrm{L}_{m i n}-\mathrm{M}_{\mathrm{H}}$ plots [39] shown by figure 21 .

But in 1992 the LHC was still foreseen for about 2000 (figure 20) and this workshop had little impact.

By the end of 1994 everything needed to predict the upper limit of $\mathrm{M}_{h}$ in the MSSM 

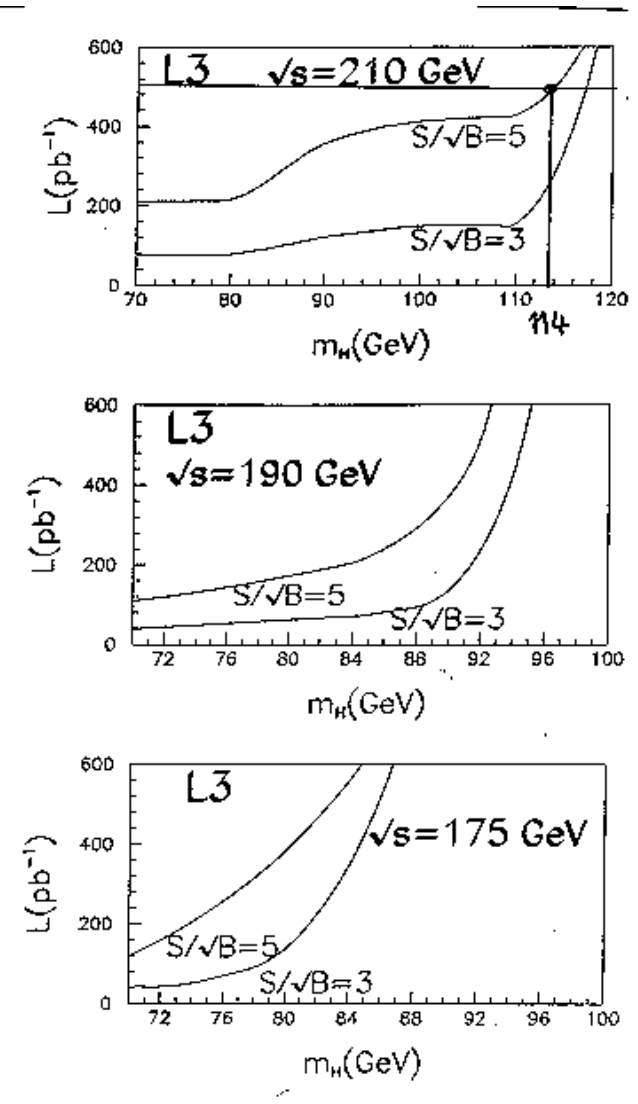

Figure 21: The Higgs discovery plot,, as shown in 1993 by J.Alcaraz et al.

was known: the top mass, as we saw, and the theoretical computation of the radiative correction to the required order (figure 22) [40], giving a figure of $\sim 126 \mathrm{GeV}$ for this upper limit, with the present central value of the top mass. The energy needed to give a meaningful answer - yes or no- about the MSSM was then well defined. From [41] one can read:

" The CM energy required to do so (i.e. complete the exploration of the MSSM "plane", $\operatorname{tg} \beta-\mathrm{M}_{\mathrm{A}}$ ) would be $\sqrt{s} \sim 210 \mathrm{GeV}(220 \mathrm{GeV})$ for $\mathrm{M}_{\text {top }} \leq 150 \mathrm{GeV}(170 \mathrm{GeV})$."

The coverage would be complete for a SM-like $\mathrm{h}^{0}$; in the general case a small tie-shaped (P.Janot's tie) domain of the plane would not be exluded.

In 1994 the LHC was approved. The foreseen date for its startup was then the end of 2002.

1995 saw the start of the energy rise at LEP, most animated by the " 4 jet" effect of Aleph.

1996 was the year of the important LEP2 workshop, which led to the Yellow Book [42], bible of the LEP200 era. By that time, improvements of the simulation and of the statistical methods were such that the crude thumb rule mass reach had become $\sim \sqrt{s}-\mathrm{M}_{Z}$ for exclusion by the four experiment added together, with typically $200 \mathrm{pb}^{-1}$ per experiment, and $\sim 2 \mathrm{GeV}$ less for discovery.

The W pair threshold was crossed in 1996. Around the same time, the production 


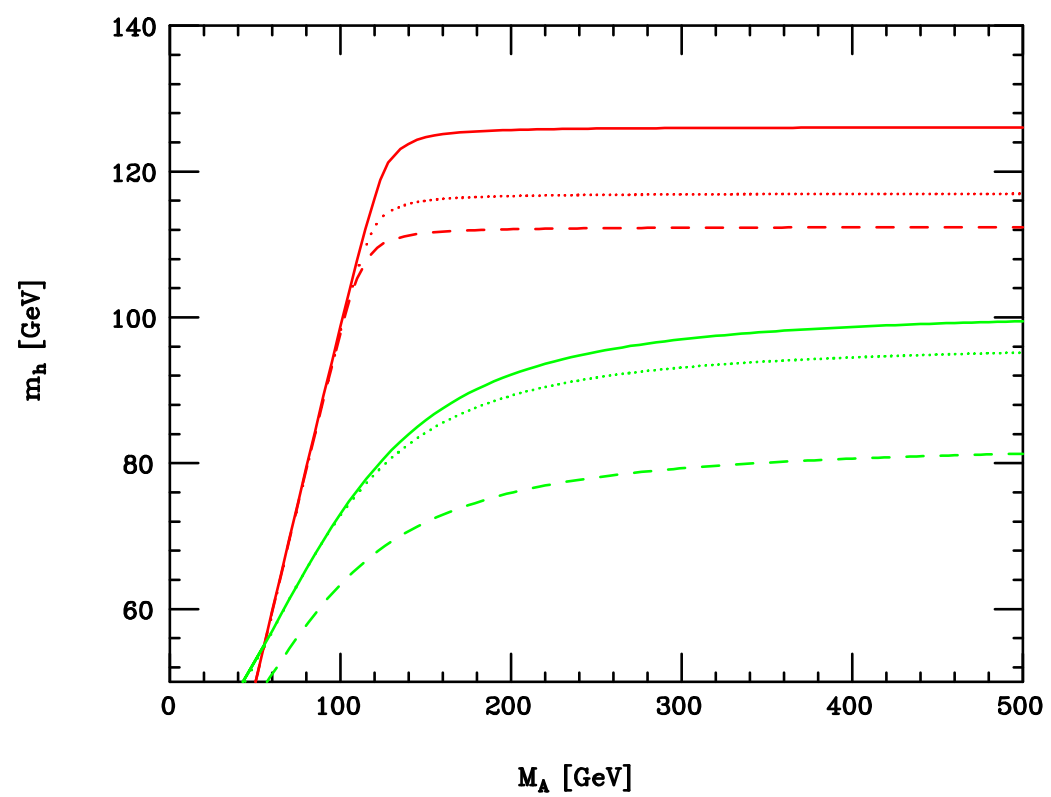

Figure 22: Upper limit of $h^{0}$ mass. Bottom curves are for small $\operatorname{tg} \beta$, upper ones for large $\operatorname{tg} \beta$.

of SC cavities was discontinued. After that you know the story, dominated by the constant progress of the machine in energy, within the allocated park of supraconducting RF cavities, as well as in luminosity, by the obtention of one extra year of running in 2000, and the difficult and painful decision to be taken at the end of 2000 .

At various occasions during the past decade, and more precisely since the goal concerning the lightest MSSM boson $\mathrm{h}^{0}$ was quantitatively settled, pleas were made to get more RF cavities in LEP, as well as to improve their accelerating field as far as possible.

The case is fairly obvious: the available CM energy goes like the fourth root of the number of RF cavities. With what was allowed to LEP (288 cavities) and the accelerating field finally achieved, LEP has reached $206 \mathrm{GeV}$. With 80 (100) cavities more, namely 365 (385), it would have reached 218 (221) GeV. This looks like a little gain. However the last mass bin of the Higgs search would have been 127 (130) GeV instead of 115 $\mathrm{GeV}$. To appreciate the impact of this fact, please look at figure 22 .

For your amusement, figure 23 quotes such an attempt, made in 1995 in a CERN Faculty meeting, as it was formulated six years ago, a time where the foreseeable competition from Fermilab came more from an hypothetical di-Tevatron than from the TeV33 option which was not yet considered. The number of cavities quoted corresponds to the maximum number which could have been installed in the even collision points, without further civil engineering, as one can read from the 96 Yellow Book [43]. The cost of such an upgrade would have represented only $\sim 3$ percent of the overall LEP project.

Unfortunately, for several reasons, including the fact that the LEP community did not really fight for it, this ultimate upgrade in the number of cavities did not happen, although a magnificent work on the LEP side gradually brought their accelerating field from 6 to $7.5 \mathrm{MeV} / \mathrm{m}$ in the niobium-film cavities which had been developed at Cern.

In 2000, as figure 24 [44] shows, the Higgs search at LEP hit the "higgsstrahlung wall", i.e. ran out of cross-section, for a mass of $\sim 115 \mathrm{GeV}$ : for a boson which would have been sitting at such a mass, the challenge was to reveal it with a production cross-section of 


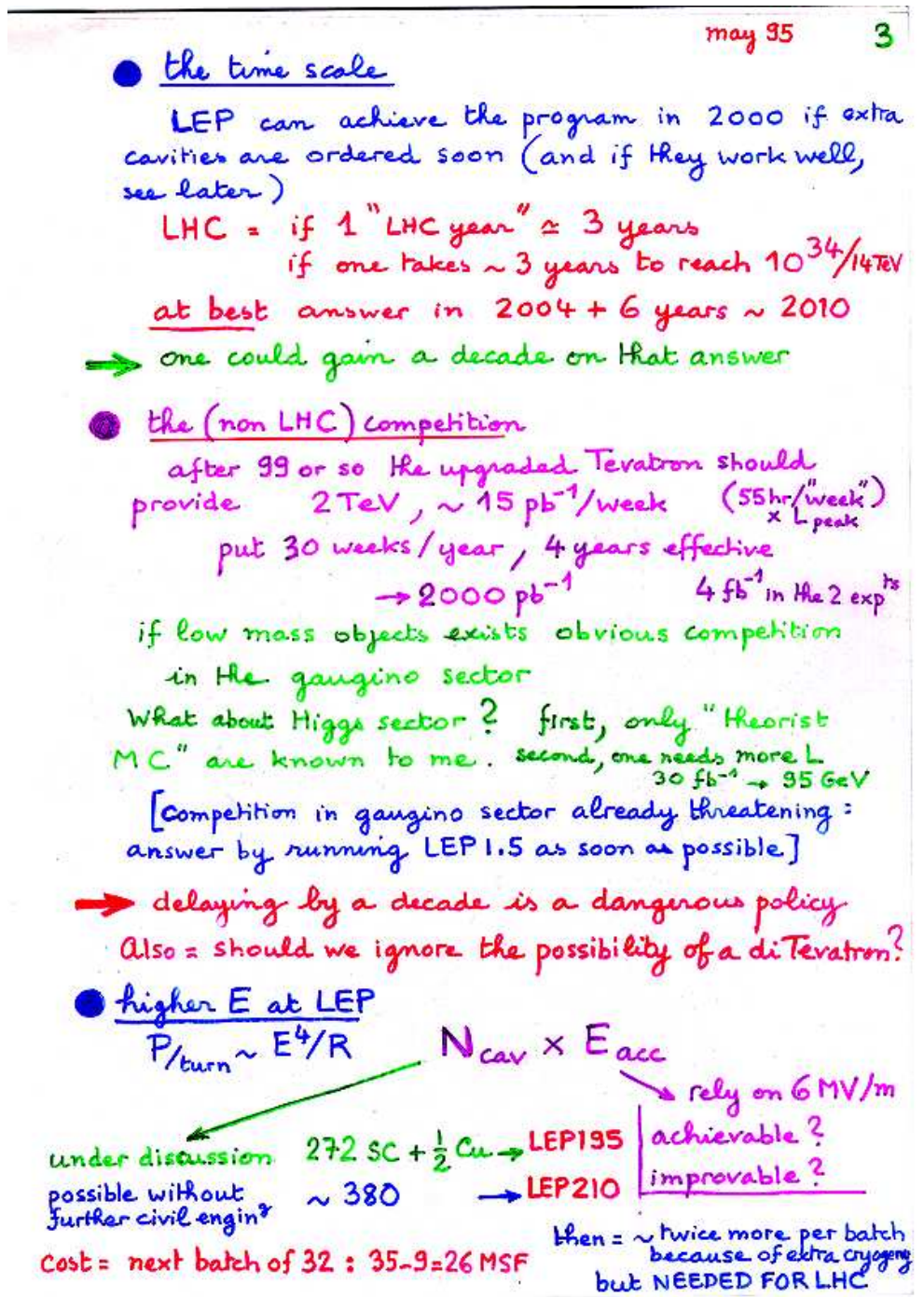

Figure 23: One of many preaches by the author....

only $50 \mathrm{fb}$, compared to $\sim 1 \mathrm{pb}$ of $\mathrm{Z}^{0} \mathrm{Z}^{0}$ and $17 \mathrm{pb}$ of $\mathrm{W}^{+} \mathrm{W}^{-}$. Other talks will give a record of the present situation of this search.

With the present limit set on the mass of a SM-like Higgs boson at $115 \mathrm{GeV}$, the status of the MSSM can be summarized by figure 25. The horizontal axis represents the mass of the lightest neutral boson $\mathrm{h}^{0}$. Each box corresponds to one of the benchmark scenarii considered by [45] and supposed to span the possibilities left open by our present knowledge. Arrows give the upper limit of $\mathrm{M}_{h}$ in the three usual SUSY breaking mechanisms. All $\mathrm{h}^{0}$ considered are perfectly SM-like. The central value of $\mathrm{M}_{\text {top }}$ has been assumed. One sees that all $\mathrm{h}^{0}$ masses foreseen fall within the reach of the gedanken LEP2, with 80/100 RF cavities more. Too bad, really too bad!..... 


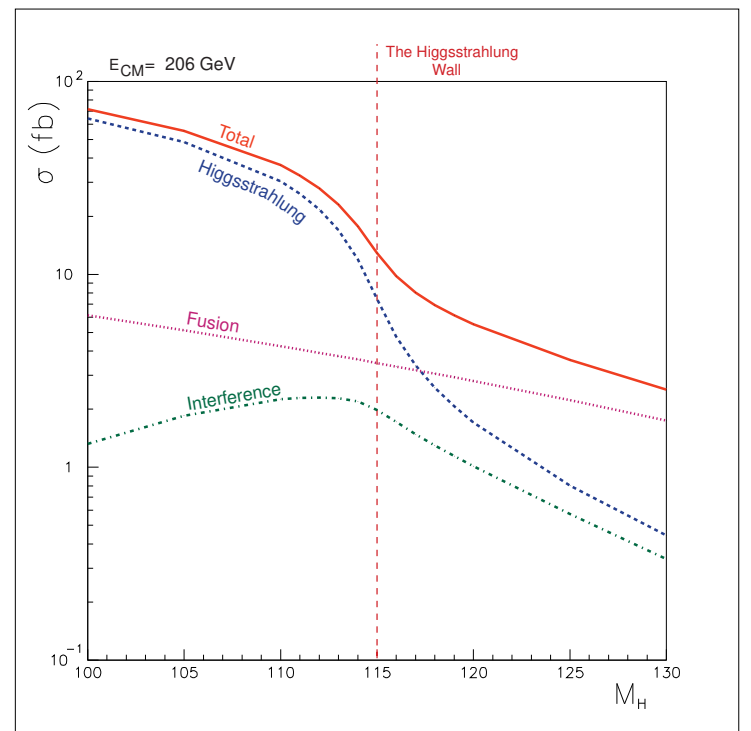

Figure 24: The cross-section for Higgs production versus its mass. Only the Z decay into neutrinos is considered.

\section{Epilogue}

The LEP era is now over, except that several analyses will still go on for some time. One should hope that a careful and clever archiving of the data will allow to interrogate them in the future if new ideas appear.

LEP and SLC have been great successes. They validated the SM at a level of accuracy which went beyond all hopes and their contributions will stay as historical landmarks. I would like to express our deep gratitude to the founders and the builders of the LEP project for their vision and their accuracy, to the machine physicists for all the miracles they have accomplished year after year, and to the many theorists whose close collaboration was so precious.

Within the SM frame and ignoring some discrepancies between observables, the

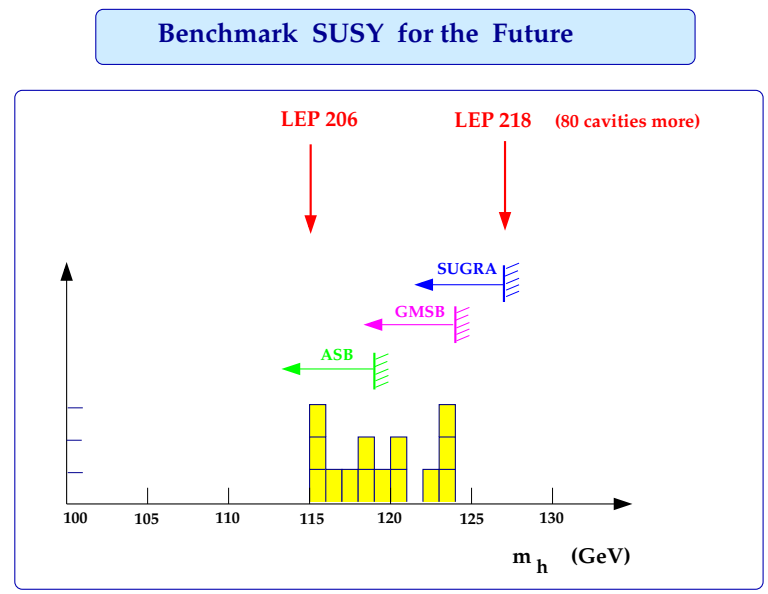

Figure 25: The MSSM benchmarks for the future. 
LEP/SLC results seem to indicate a light Higgs boson. The convergence of couplings seems to favour a supersymmetric solution. Both facts, put together, go in the direction of a MSSM type of theory.

The smoking gun of MSSM (the only feature one can be sure about) is the existence of a quite light neutral higgs boson. Unfortunately, whatever point of view one may have on the 2000 Higgs Odyssey, LEP has not brought an answer to that crucial question. We only know that a SM like neutral Higgs boson, if any, has a mass $\geq 115 \mathrm{GeV}$.

One could say that, thanks to this ignorance, the motivation for the future enterprises is preserved. I will not follow such an argumentation which sits beyond the border of any scientific attitude. On the contrary I find that the situation is in many respects very regrettable. From the Tevatron or LHC, which program will answer first about the existence of a light object is an open question. Let us hope that the additional pressure put on LHC by this competition will not be counterproductive.

Acknowledgments: I am very grateful to the organizers of the meeting, held in such a wonderful area, for offering me this talk. I thank Luc Pape and Felicitas Pauss for many discussions and comments.

\section{References}

[1] M.Davier and A.Hocker, LAL97-85, hep-ph/9711308

[2] These figures, as well as several quotations appearing below, are borrowed from W.Venus' talk in Budapest.

[3] M.E.Peskin, Theoretical Summary Lecture for EPS HEP99, SLAC-PUB-8351, february 2000, hep-ph/0002041.

[4] C.Quigg, Top-ology, Physics Today 50 (5) 1997, 20-26, hep-ph/9704332.

[5] M.E.Peskin and J.D.Wells, SLAC-PUB-8763, LBNL-47226, Jan 2001.

[6] B.Pietrzyk, private communication.

[7] U.Amaldi et al, Phys.Lett. B260 (1991) 447, and references therein.

[8] H.Videau, private communication.

[9] F.Caravaglios et al, LAL-00-04.

[10] B.Richter, CERN/ISR-LTD/76-9, march 1976.

[11] I am told the figure was drawn and the name Crazytron coined by G.Voss.

[12] Blue Book, CERN/ISR-LEP/78-17, Pink Book, CERN/ISR-LEP/79-33, LEP Design Report, CERN/LEP/84-01.

[13] Les Houches LEP Summer Study, CERN 79-01, feb. 1979. 
[14] Physics at LEP, edited by John Ellis and Roberto Peccei, CERN 86-02, february 1986.

[15] B.W.Lynn, M.E.Peskin and R.G.Stuart, page 90 of ref.14.

[16] The LHC option, ECFA Workshop in march 1984, Cern Courier, july/august 1986.

[17] La Thuile 1987, CERN 87-07.

[18] C.Prescott, SLAC-PUB-6987, aug 1995, SLAC-PUB-6242, july 1993.

[19] Polarization at LEP, CERN 88-06, sept 88,edited by G.Alexander, G.Altarelli, A.Blondel, G.Coignet, E.Keil, D.E.Plane and D.Treille.

[20] A.A.Sokolov and I.M.Ternov,Sov. Phys. Dokl. 8 (1964) 1203.

[21] A.Blondel, Phys.Lett. 202B (1988) 145.

[22] R.Schwitters and B.Richter, SLAC PEP Note 87 (1974).

[23] Results in SLAC-PUB-8401, PRL 84:5945-5949, 2000.

[24] High Luminosities at LEP, CERN-91-02, march 1991, edited by E.Blucher, J.Jowett, F.Merritt, G.Mikenberg, J.Panman, F.M.Renard and D.Treille.

[25] C. de la Vaissiere et al, CERN/DELPHI 89-5 Phys 5 (1989).

[26] B decays, p166 of ref.24.

[27] Contributions by P.Roudeau, C.Defoix, H.G.Moser, in ref.24.

[28] E.Longo, in ref.19, vol.1, p.304.

[29] G.Feldman, MkII/SLC P.W.G. note 2-24, 1987.

[30] E.Lieb et al, ref.24, p.279.

[31] Talk by Ugo Amaldi in Cogne, for the Delphi Collaboration. In Cern Archives.

[32] The names of G.von Holtey, P.Roudeau and D.Ritson are associated to these studies.

[33] B.Pietrzyk, LAPP-EXP-94-18, Oct 1994, and references therein.

[34] J.M.Heuser, Nucl.Instrum.Meth.A418:1-8,1998, and references therein.

[35] ECFA Workshop on LEP200, Aachen, 29 sept-1 oct 1986, CERN 87-08, ECFA 87/108, june 1987, editors: A.Bohm, W.Hoogland. The paper on SC RF is by Ph.Bernard, H.Lengeler and E.Picasso, vol.1, p.29.

[36] Sau Lan Wu et al, p.312 of ref.35.

[37] D.Treille, CERN-EP/90-30, march 1990. 
[38] Y.Okada, M.Yamaguchi and T.Yanagida, Prog.Theor.Phys. 85 (1991), H.E.Haber and R.Hempfling, PRL. 66 (1991) 1815, J.Ellis, G.Ridolfi and F.Zwirner, Phys.Lett.B 257 (1991) 83.

[39] These plots are associated to P.Janot's name. The plot of figure 21 is taken from J.Alcaraz et al, Cern-PPE/93-28.

[40] M.Carena et al, Phys.Lett.B355; 209-221,1995, hep-ph/9504316.

[41] D.Treille, Searches for New Particles, CERN-PPE/94-114, and Rep.Prog.Phys 57 (1994) 1137.

[42] Physics at LEP2, CERN 96-01, february 1996.

[43] S.Myers and C.Wyss, in ref.42, vol.1, p.23.

[44] E.Gross and A.L.Read, CERN-EP-2000-034, Feb 2000. See also P.Janot, in Rencontres de Blois, June 27-July 3, 1999.

[45] M.Battaglia et al, CERN-TH-2001-150, UMN-TH-2013-01, TPI-MINN-01-29, June 2001. 